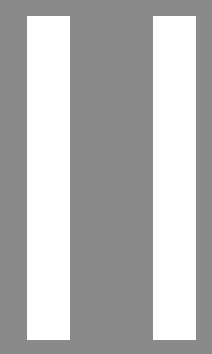

\title{
COMPETENCIAS PROFESIONALES REQUERIDAS POR LAS EMPRESAS EN TARAPOTO
}

\section{Professional skills required by companies in Tarapoto Universidad Peruana Unión, Perú}

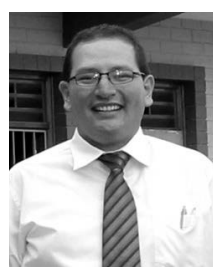

\section{Mario Manuel Siles Nates}

Ingeniero comercial y licenciado en Administración de Empresas por la Universidad Católica Santa María, Perú. Magíster en Economía por la Universidad Nacional San Agustín de Arequipa, Perú. Doctorando en Administración con mención en Gestión y Dirección de Organizaciones por la Universidad Peruana Unión. Docente asociado de la Facultad de Ciencias Empresariales de la Universidad Peruana Unión. Exgerente de ventas de Productos Unión. Actualmente se desempeña como director y coordinador de la Facultad de Ciencias Empresariales de la Universidad Peruana Unión, con sede en Tarapoto. 


\section{Resumen}

El presente artículo tiene como propósito presentar, de manera descriptiva, los resultados del estudio sobre las competencias profesionales de mayor demanda en la ciudad de Tarapoto, San Martín, Perú; realizada en 108 empresas, asimismo, desarrollar un análisis por sectores de las empresas que están adscritas a la Cámara de Comercio de San Martín. Estas competencias fueron agrupadas en cinco categorías: competencias de gestión personal, de gestión de trabajo en equipo, de influencia, cognitivas, de logro y práctica de valores. Entre los resultados se encuentra la supremacía de las competencias del trabajo en equipo que agrupan a las competencias, solución de problemas, liderazgo, empatía, naturalidad, y trabajo en equipo. Seguida de la práctica de los valores y las competencias de influencia. Siendo estos los tres agrupamientos de mayor priorización y demanda en los profesionales que laboran actualmente en las mencionadas empresas.

Palabras clave: Competencias profesionales, gestión personal, talento humano.

\section{Abstract}

This article aims to present a descriptive survey results on skills most in demand in the city of Tarapoto, San Martin, Peru, conducted in 108 companies also develop a sectoral analysis of the companies that are attached to Chamber of Commerce of San Martin. These competencies were grouped into five categories: personal management skills, management, teamwork, influence, cognitive achievement and values, practice of values. Among the results is the supremacy of teamwork skills that group skills, problem solving, leadership, empathy, naturalness, and teamwork. Followed by the practice of the values and skills of influence. These being the three largest clusters demand prioritization and professionals working in the mentioned companies currently.

Key words: Professional skills, personnel management, human talent. 


\section{Introducción}

El presente trabajo de investigación tiene por objeto determinar las competencias profesionales de mayor demanda en las empresas de Tarapoto.

Siendo la primera variable a estudiar las "Competencias" y apoyados en los estudios desarrollados por Mc Clelland (1973) y Spencer y Spencer (1993); la consultora HAYGROUP clasifica las competencias en cinco grandes grupos que constituyen nuestras variables de estudio:

- Competencias de gestión personal.

- Competencias de gestión de equipo de trabajo.

- Competencias de influencia.

- Competencias cognitivas.

- Competencias de logro.

Además se decidió incorporar un grupo aparte denominado Valores producto de la mezcla cultural, las tradiciones y el entorno en el cual viven los colaboradores y empresarios que formaron parte del presente estudio para poder separar claramente los grupos anteriores.

En ese sentido delimitando el entorno de estudio, Chiavenato (2009), afirma que las organizaciones no funcionan sin los individuos y estos no viven sin ellas; haciendo de esta relación diádica de vital importancia para el equilibrio de satisfacciones que genera la empresa a los cinco (5) actores vinculados a ella como son: los consumidores, proveedores, dueños, sociedad y los trabajadores; con ello el mencionado autor afirma que la competitividad de las empresas se fundamentan en los colaboradores competitivos con los que cuentan. Por ello el tener especial cuidado al integrar a los talentos a la organización se requiere, para esta tarea, mayor atención y especialización.

En esa línea de estudio Alles (2008), afirma que si una persona tiene capacidades naturales, estas pueden ser potenciadas o anuladas según sus conductas, partiendo que las competencias que posee un profesional agrupa las destrezas, aptitudes y conocimientos requeridos para ejecutar la profesión y que estas son potencializadas con el comportamiento cotidiano o su personalidad. Con ello hoy la neuroselección de personal se perfila como uno de los avances en el campo de la Gestión del talento humano, siendo aún un método experimental.

Flores (2009), menciona que es conocido que las empresas desarrollan su modelo organizacional y también determinan la estructura, desarrollando 
el perfil del puesto en el cual también aparecen las competencias necesarias que se requiere de los colaboradores. Además, al citar a Claux, Luyo, Solano y Young (2001), en la investigación realizada por la bolsa de trabajo de la Pontificia Universidad Católica del Perú, de la autora citada al inicio del párrafo, reporta que las competencias que priorizan las empresas son el trabajo en equipo, las habilidades de comunicación y la creatividad e innovación, siendo que las empresas de rubros como banca, telecomunicaciones, producción de bienes masivos, tecnología, seguros y consultoría y todas estas empresas consolidadas en la ciudad de Lima.

Figura1. Producto Bruto Interno (PBI) trimestral: 2004- I 2013 - I

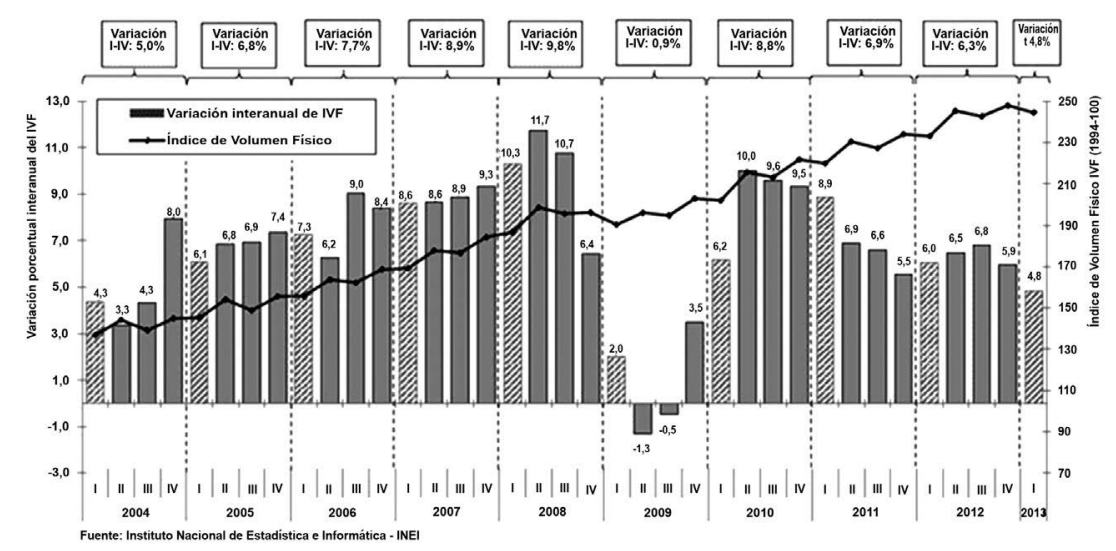

Tomando en cuenta el contexto de las empresas, vemos el sostenido crecimiento de la economía peruana en la última década que muestra un ritmo del $6 \%$, observando el compromiso del empresariado peruano por la exportación y la función del emprendedorismo como parte del impulso empresarial; asimismo, se observa el esfuerzo de los actores públicos y privados por promover la oferta exportable, la investigación comercial y la cultura exportadora, como se observa en la Figura1. 
Tabla 1. Distribución de PEA ocupada en el Perú / según tamaño de empresa y estructura de mercado (2002).

\begin{tabular}{lrrr}
\hline Categoría & PEA Ocupada & PEA Ocupada Urbana & PEA Ocupada Rural \\
\hline Independiente (auto & & & \\
empleado) & $2,512,603$ & $1,411,281$ & $1,104,322$ \\
Microempresas & $6,352,695$ & $3,387,651$ & $2,965,044$ \\
Pequeñas empresas & 873,687 & 739,416 & 134,271 \\
Medianas y grandes & & & \\
empresas & 975,429 & 886,407 & 89,022 \\
Otros & $1,355,658$ & $1,165,041$ & 190,617 \\
Total & $12,070,072$ & $7,589,796$ & $4,483,276$ \\
\hline
\end{tabular}

Fuente: Lévano (2005), Ministerio de Trabajo y Promoción del Empleo (2004). *Incluye trabajadores del sector público y trabajadores del hogar.

Lévano (2005), afirma que las microempresas constituían más del noventa y siete por ciento (97\%) del total de la distribución de empresas en el país, además eran las que ocupaban a más de la mitad (52\%) de los peruanos dentro de las mismas.

Del mismo modo Villarán (2007), en su libro “El mundo de la pequeña empresa", se esmera en presentar un diagnóstico y critica la situación de la micro y pequeña empresa en el Perú, refuerza la idea de que las microempresas son impulsoras del empleo y que aún muestran resistencia en pasar a la formalidad, con ello es necesario plantear reformas proclives a mejorar la situación de su formalización sin la asfixiante recaudación tributaria, mejor manejo del proceso de formalización y mayor acceso al crédito formal. Resaltando a los actores principales de creación de valor, los que hacen que la microempresa se mueva, como sus colaboradores.

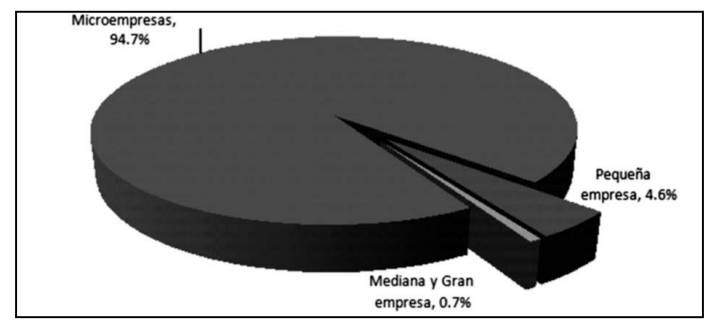

Fuente: Superintendencia Nacional de Administración Tributaria SUNAT Elaboración: PRODUCE - DGMYPE /dirección regional empresarial

Figura 2. Distribución de las empresas formales en el Perú 
Analizando la estructura de generación de empleo actual en el Perú, se puede observar el predominio que mencionaron los autores anteriores; la microempresa; Fereyros Gerente de Comex Perú, en el año 2012, afirmó que la mediana y pequeña empresa aportan con el $42 \%$ de la producción total en el Perú y son generadoras de empleo de casi 13.3 millones de personas mostrando una vez más el rol indiscutible que tiene la pequeña empresa en el desarrollo social y económico del Perú.

Se debe de agregar que el estudio desarrollado por el Ministerio de la Producción a fines del 2012, como se puede apreciar en la Figura 2, el 94.7\% son microempresas, el $4.6 \%$ son pequeña empresa y el $0.7 \%$ son mediana y gran empresa de un total de 1'200,664 empresas formalmente constituidas. Por otro lado, más del $80.9 \%$ son empresas unipersonales, además se puede acotar que en su mayoría se encuentran en el sector comercio (37\%); por otro lado, en su distribución, a nivel nacional, se denota una concentración del 48.7\% de las Mypes en Lima ciudad capital, además existen 7 regiones dinámicas que concentran el $28.8 \%$ del restante grupo de microempresas como se observa en la Figura 3

La región San Martín solo cuenta con el $1.7 \%$ del total de la distribución de las MYPES, siendo esta región unas de las siete (7) regiones con menor acumulación de MYPES formales, toda esta información es parte de la memoria anual de Ministerio de la Producción quienes, en un denodado esfuerzo, resaltan el crecimiento de la formalidad en los últimos 3 años debido a los programas diseñados por parte del gobierno con la misión de reducir la brecha fiscal existente en nuestro país.

En la región San Martin, donde es el campo de trabajo de la presente investigación, la estructura económica se basa en las actividades agropecuarias, agroindustriales, turísticas y comerciales que son las que impulsan el motor económico de la región. Las líneas de transformación en el cual se desempeñan los microempresarios son la carpintería, metalmecánica, ladrillera, industrias del vestido, fabricación de postes, la industria del tabaco y artesanías, siendo las más dinámicas la carpintería y las confecciones. Datos recolectados del plan de desarrollo urbano de Tarapoto. 


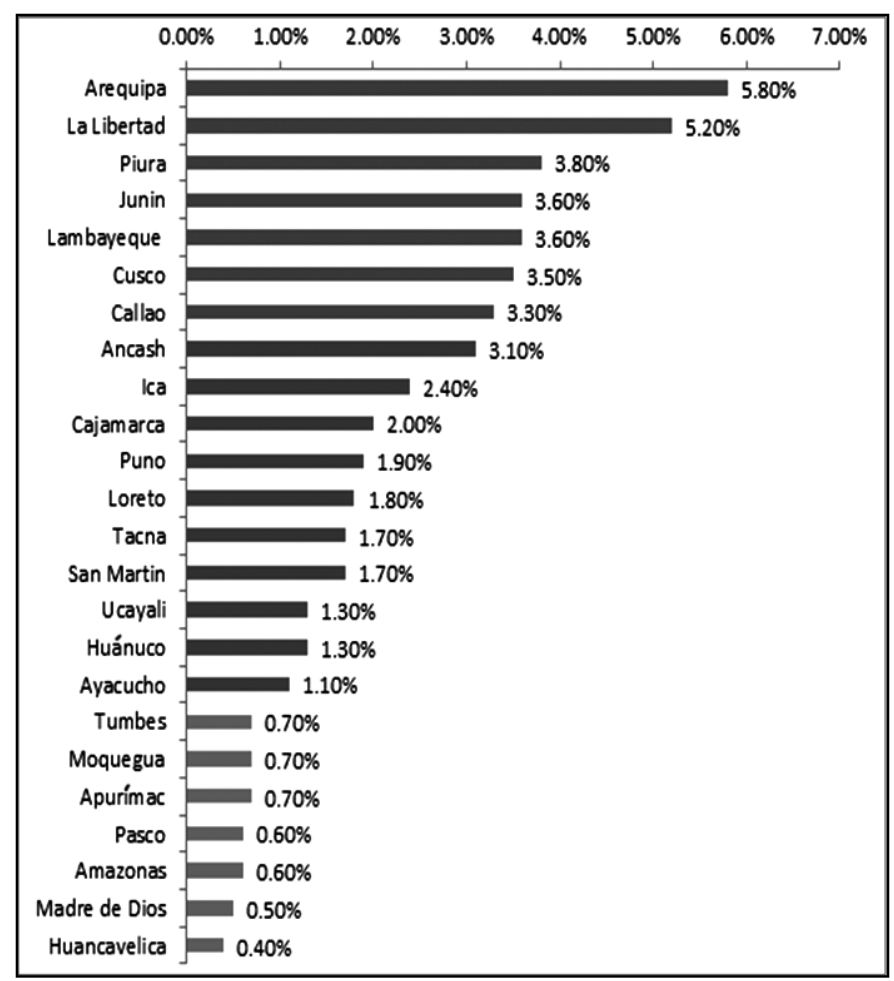

Fuente: SUNAT

Elaboración: PRODUCE - DGMYPE /dirección regional empresarial

Figura 3. Distribución de las Mypes por regiones sin contar Lima

Se debe mencionar que existen problemas "neonatológicos" en los emprendimientos formales de la región, donde el nacimiento es muy rápido con grandes expectativas, pero la vida empresarial es muy corta sin llegar a la consolidación, solo del $2 \%$ al $4 \%$ de todos los emprendimientos llegan a los 3 años de vida, siendo el sector comercio el que agrupa a la mayor cantidad de microempresas.

La preocupación por la determinación de las principales competencias requeridas solicitadas por las empresas en Tarapoto que como vimos en su mayoría son pequeñas y micro, se hace relevante en dos ámbitos, corres- 
pondiendo a la empresa el conocer cuáles son las competencias develará los puntos en los cuales basa la competitividad de cada empresario y por el lado académico superior, dado que son las universidades e institutos los que lideran la formación en la región y el monitoreo constante del mercado laboral ayudarán a mejorar los perfiles de los egresados y con ello una preparación competente en las áreas de interés de los demandantes directos e indirectos del mercado laboral y mejorar el perfil emprendedor de las carreras de la gestión empresarial.

Entre los objetivos de este estudio se encuentran:

- Identificar las principales competencias solicitadas por las empresas.

- Conocer las técnicas de selección de personal utilizadas por las empresas.

- Identificar las principales técnicas de reclutamiento de personal utilizadas por las empresas.

El tipo de investigación es descriptiva de corte transversal, el ambiente en el cual se desarrolló fue la ciudad de Tarapoto, en el departamento de San Martín, Perú. (Hernández, Fernández y Baptista, 2010).

\section{Método}

La unidad de Estudio utilizada fueron las empresas que desarrollan sus actividades en el ámbito de la ciudad de Tarapoto, dentro de la circunscripción de los tres distritos: Morales, La Banda de Shilcayo y Tarapoto. La unidad reportante fue el administrador o encargado del área de Recursos Humanos de las medianas, pequeñas y microempresas de Tarapoto.

Los criterios de inclusión utilizados fueron:

a) Empresas hábiles y registradas de la Superintendencia Nacional de Administración Tributaria (SUNAT).

b) Tres (3) años consecutivos e ininterrumpidos de funcionamiento.

c) Adscritas a la cámara de comercio de San Martín.

d) Que se encuentren funcionando en los distritos Morales, Banda de Shilcayo y Tarapoto.

Para la determinación de la muestra, se ejecutó la fórmula propuesta por Munich y Ángeles (1996), en Métodos y técnicas de investigación para 
poblaciones finitas (citado por Valderrama, 2002). La que se utiliza para calcular el tamaño de la muestra en trabajos de investigación educacional y en ciencias sociales.

$$
n=\frac{N * Z^{2} * p^{*} q}{(N-1) * e^{2}+Z^{2} * p^{*} q}
$$

En donde:

Z= Nivel de confianza (1,96 tabla de distribución normal para el 95\% de confiabilidad)

$\mathrm{N}=$ Población (180)

$\mathrm{p}=$ Probabilidad a favor $(0.50)$

$\mathrm{q}=$ Probabilidad en contra $(0.50)$

e= Error de estimación $(6 \%=0.06)$

$\mathrm{n}=$ Tamaño de la muestra

$$
\begin{aligned}
& n=\frac{180 * 1,96^{2} * 0,5 * 0,5}{(180-1) * 0,06^{2}+1.96^{2} * 0,5 * 0,5} \\
& n=\frac{173.83}{1.60} \\
& n=108
\end{aligned}
$$

Teniendo como resultado ciento ocho (108) empresas seleccionadas, además se mantuvo la proporcionalidad de cada sector en la muestra asegurando, de esta manera, que el modelo se asemeje a la realidad.

Se utilizó el muestreo probabilístico estratificado citado por Luque (1994), donde se lo define como "el muestreo que consiste en dividir la población en " $\mathrm{n}$ " subconjuntos o estratos, y de cada uno de ellos seleccionar una muestra probabilística; de manera independiente de un estrato a otro".

Para la elección dentro de cada estrato se utilizó el muestreo aleatorio simple, tomando como base la lista de ciento ocho (108) empresas que cumplieron los criterios antes mencionados, como se aprecia en la Tabla 2.

Tabla 2. Empresas por sectores adscritas a la Cámara de Comercio de San Martín (CCSM), población de estudio y la muestra por cada estrato. 
Mario Manuel Siles Nates

\begin{tabular}{|c|c|c|c|c|c|c|}
\hline \multirow[b]{2}{*}{ Sectores } & \multicolumn{2}{|c|}{ Empresas adscritas a CCSM } & \multicolumn{2}{|c|}{ Población de Estudio * } & \multicolumn{2}{|c|}{ Muestra del Estudio } \\
\hline & $\begin{array}{l}\text { Número } \\
\text { de empresas }\end{array}$ & Porcentaje & $\begin{array}{l}\text { Número } \\
\text { de empresas }\end{array}$ & $\begin{array}{l}\text { Porcen- } \\
\text { taje }\end{array}$ & $\begin{array}{l}\text { Número } \\
\text { de empresas }\end{array}$ & $\begin{array}{l}\text { Porcen- } \\
\text { taje }\end{array}$ \\
\hline Comercio & 81 & (33.61) & 77 & $(42.78)$ & 46 & $(42.59)$ \\
\hline Servicios & 76 & $(31.54)$ & 63 & $(35.00)$ & 38 & (35.19) \\
\hline Producción & 50 & $(20.75)$ & 22 & $(12.22)$ & 13 & $(12.04)$ \\
\hline Turismo & 34 & $(14.11)$ & 18 & $(10.00)$ & 11 & $(10.19)$ \\
\hline Total & 241 & $(100)$ & 180 & $(100)$ & 108 & $(100)$ \\
\hline
\end{tabular}

* Empresas que cumplen con los cuatro criterios de inclusión.

Fuente: Cámara de Comercio de San Martin CCSM - SUNAT sede Tarapoto.

Elaboración: Propia

\section{Instrumento}

Sobre el cuestionario: cuenta con tres secciones claramente delimitadas; en la primera sección se agrupan los datos generales de la empresa: razón social, sector, número de trabajadores siendo estas las variables de control de parte del presente estudio.

La segunda sección consta de siete (7) preguntas sobre las competencias que son demandadas por las organizaciones. La pregunta 1 es una escala comparativa de clasificación, donde se pretende observar el ordenamiento en función a la importancia, que los responsables de la empresa otorgan a los cinco (5) agrupamientos de competencias.

Tomando como referencia los trabajos de Mc Clelland 1973 y Spencer y Spencer (1993), la consultora HAYGRUP pionera en la implantación de gestión por competencias en las empresas clasifica las competencias en cinco grupos: gestión personal, trabajo en equipo, de influencia, cognitivas, de logro y los valores provenientes de la parte sociocultural.

Las preguntas 2 a la 7 son preguntas de opción múltiple, observando las preferencias de las competencias que se encuentran incluidas dentro de los cinco (5) agrupamientos con las indicaciones de seleccionar como mínimo tres (3) y máximo cinco (5) en las opciones presentadas en cada agrupamiento de competencias.

La tercera sección consta de seis (6) preguntas y centra su atención en el área de gestión del talento humano, la pregunta 9 precisa información 
sobre la carrera profesional del responsable del área, el género y el número total de personas que laboran a dedicación del área de Recursos Humanos.

Las preguntas 10 y 11 son preguntas de opción múltiple y determinan las preferencias sobre las técnicas habitualmente utilizadas en el proceso de reclutamiento y selección con las indicaciones de seleccionar como mínimo tres (3) y máximo cinco (5) entre las opciones presentadas en cada agrupamiento de competencias.

\section{Confiabilidad}

Se desarrolló una encuesta piloto de 15 empresas, probando claridad y precisión en la preguntas.

Para la validación de instrumento, se recurrió al juicio de los expertos, siendo validado por el Dr. Luis Vargas Espinoza, docente asociado de la Universidad Católica de Santa María adscrito a la Facultad de Ciencias Económico - administrativas, así también por el Dr. Jhon Loayza Borja, docente principal adscrito a Escuela de Sociología de la Universidad Nacional de San Agustín Arequipa, quienes evaluaron la pertinencia y la adecuación del cuestionario.

En segundo lugar, se procedió a analizar la confiabilidad, mediante la técnica estadística del Alfa de Cronbach, utilizando en el paquete estadístico SPSS 20, la cual es una técnica que mide la consistencia interna del cuestionario proporcionado el coeficiente de 0.8 considerado un coeficiente aceptable según Hernández, Fernández y Baptista (2010), como se aprecia en la Tabla 3.

Tabla 3. Estadístico Alfa de Cronbach de la encuesta de principales competencias

\begin{tabular}{ccc}
\hline Cuestionario & Alfa de Cronbach & N. $^{\circ}$ de elementos \\
\hline Principales competencias & 0.798 & 68 \\
\hline Elaboración: Propia & &
\end{tabular}

\section{Resultados}

Análisis descriptivo de los resultados

En la figura 4 se puede apreciar las competencias de mayor importancia para las medianas, pequeñas y microempresas de la ciudad de Tarapoto; siendo las relacionadas al "Trabajo en equipo" con un $40.74 \%$, las mismas que 


\section{Mario Manuel Siles Nates}

comprenden las competencias de trabajo en equipo, solución de problemas, liderazgo, empatía, naturalidad, dirección y desarrollo de personas.

Figura 4. Competencias consideradas de mayor importancia por todos los sectores empresariales inscritos en la CCSM Tarapoto

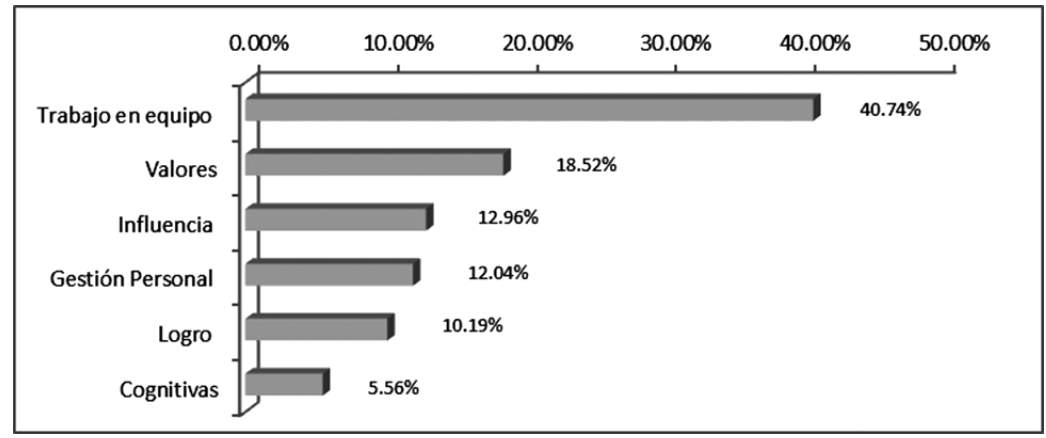

En segundo lugar se encuentran los valores con un $18.52 \%$, siendo los más relevantes: la honradez, puntualidad, conducta ética profesional con lo se puede inferir que el empresario valora, de sus colaboradores, la forma de lograr los objetivos organizacionales mediante el esfuerzo cooperativo y la práctica de los valores que, como ya explicamos anteriormente, son resultados de todos los que trabajan.

Figura 5. Técnicas de reclutamiento de personal utilizado por las empresas inscritas en la CCSM Tarapoto

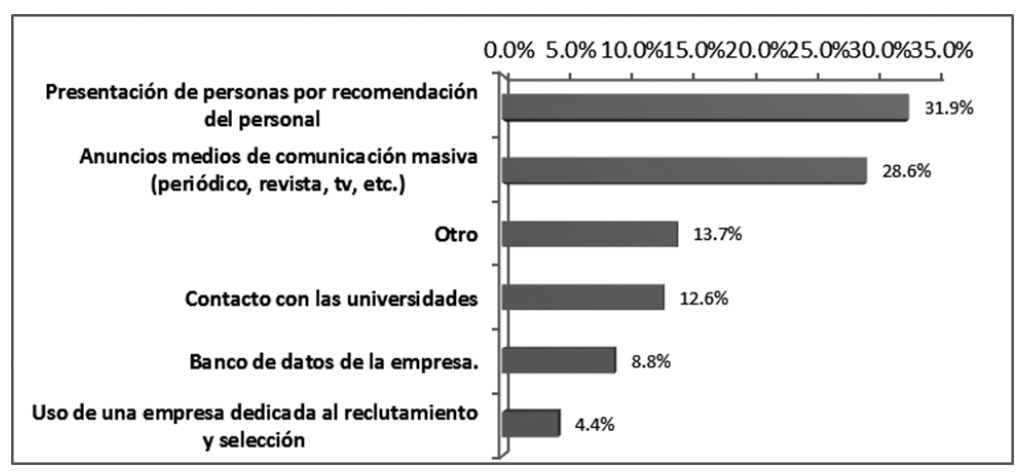


En la figura 5 se puede observar que utilizan "la recomendación de personal que se encuentra laborando actualmente con ellos" en un $31.9 \%$; se infiere que es una forma cuyo costo es menor y busca utilizar la palabra empeñada del recomendado como punto de referencia y fuente fidedigna de información sobre las competencias de su recomendado, en función de los requerimientos del puesto a ocupar.

Como punto a favor de este método se menciona que el personal que labora en las microempresas tiene conocimiento sobre el clima laboral, el proceso administrativo, el estilo de liderazgo y con ello informa de alguien que pueda ocupar el puesto solicitado. No dejando de lado que este método es experimental no profesional.

Anuncios por medios de comunicación masiva como radio, televisión, periódico tienen un $28.6 \%$, haciendo masiva la convocatoria de este manera. Seguido de $13.7 \%$ de otras técnicas como el recomendación de amigos y familiares, anuncios en la fachada del local y, por último, difundir la noticia en el Facebook de la empresa como enlace.

Figura 6. Técnicas de selección de personal utilizado por las empresas inscritas en la CCSM Tarapoto

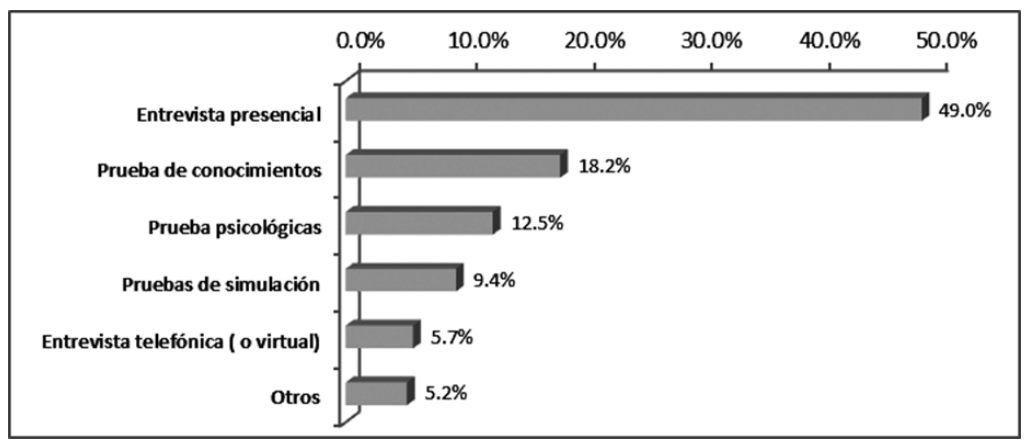

Como se puede apreciar, la entrevista personal (49\%) la utiliza como herramienta preferida para seleccionar a su personal; seguido de las pruebas de conocimientos (18.2\%), pruebas psicológicas (12.5\%) siendo estas últimas solo en las empresas que tercerizan el proceso de reclutamiento y selección.

A continuación se presentan las tablas de los distintos sectores y las competencias de competencias, contenidos en el presente trabajo.

El sector comercio es el sector que agrupa a más del $42 \%$ del total 
de la muestra del presente estudio, muestra que el $41.3 \%$ de las empresas de Tarapoto priorizan las competencias de trabajo en equipo, seguidas de las competencias de influencia y los valores en el segundo lugar con el $15.22 \%$ como se muestra en la Tabla 4.

Tabla 4. Principales competencias valoradas por las empresas agrupadas en el sector comercio

\begin{tabular}{lcc}
\hline \multicolumn{1}{c}{ Competencias } & Recuento* & Porcentaje \\
\hline Trabajo en equipo & 19.00 & $(41.30)$ \\
Influencia & 7.00 & $(15.22)$ \\
Valores & 7.00 & $(15.22)$ \\
Logro & 6.00 & $(13.04)$ \\
Gestión Personal & 5.00 & $(10.87)$ \\
Cognitivas & 2.00 & $(4.35)$ \\
Total & 46 & 100.00 \\
\hline
\end{tabular}

* Empresas solo del sector de comercio que cumplen con los requisitos de inclusión

En las técnicas de reclutamiento de personal vemos que el 31.9\% del total aplica la recomendación de los trabajadores como técnica de reclutar, seguido de los anuncios por medios masivos con un $30.6 \%$ y los carteles en las tiendas y el recomendaciones de familiares y amigos en tercer lugar $15.3 \%$, datos que se pueden ver en la Figura 7.

Figura 7. Técnicas de reclutamiento de personal utilizado por las empresas inscritas en la CCSM del sector comercio en Tarapoto

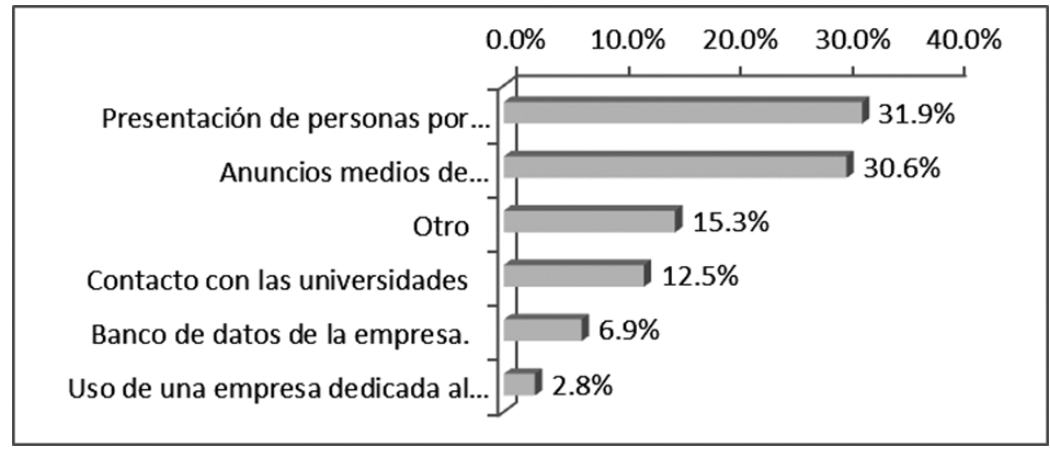


La técnicas de selección de personal que se aplican en el sector comercio de Tarapoto se pueden apreciar en el la figura 8, donde la entrevista personal es una de las técnicas más utilizadas con el $46.5 \%$, dado que es necesario conocer al candidato y el tema de la primera impresión también les es importante.

Figura 8. Técnicas de selección de personal utilizadas por las empresas inscritas en la CCSM del sector comercio en Tarapoto

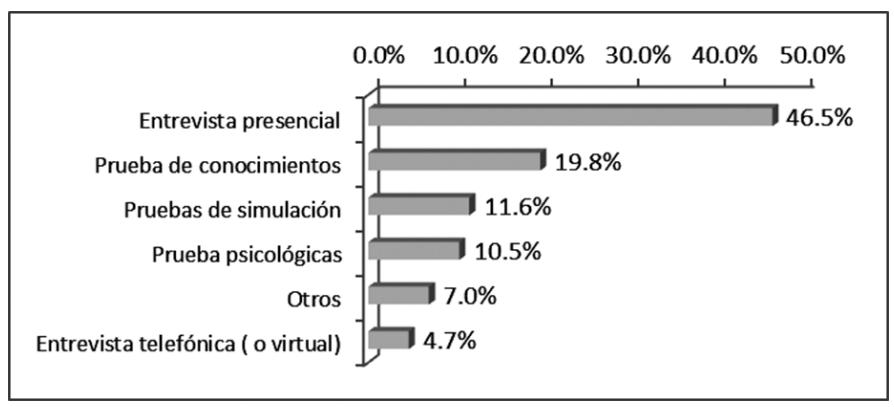

En segundo lugar, las pruebas de conocimientos en un $19.8 \%$, las pruebas de simulación con un $11.6 \%$, estas últimas desarrolladas posterior a la entrevista. Asimismo solo un $10.5 \%$ utiliza pruebas psicológicas dado que en la ciudad solo existen tres empresas dedicadas al rubro y que cuentan con profesionales en el área de psicología dedicados a desarrollar el mencionado trabajo.

El sector producción está conformado por manufactura, sector de la construcción, la porcicultura, agricultura e industria y dado que algunas de las empresas no cumplían los criterios de inclusión por el lugar de estudio y los años, encontramos una menor concentración de empresas en este rubro.

Tabla 5. Principales competencias valoradas por las empresas agrupadas en el sector Producción

\begin{tabular}{lcc}
\hline Competencias & Recuento* & Porcentaje \\
\hline Trabajo en equipo & 7 & $(53.85)$ \\
Cognitivas & 2 & $(15.38)$ \\
Valores & 2 & $(15.38)$ \\
Gestión Personal & 1 & $(7.69)$ \\
Logro & 1 & $(7.69)$ \\
Influencia & 0 & $(0.00)$ \\
Total & 13 & $(100)$ \\
\hline
\end{tabular}

* Empresas solo del sector de Producción que cumplen con los requisitos de inclusión 
Siendo el $12 \%$ del total de la muestra, como se puede apreciar en la Tabla 5, las competencias de trabajo en equipo lideran la valoración de los empresarios con un $53.85 \%$, seguido de las competencias cognitivas y los valores con un $15.38 \%$.

Figura 9. Técnicas de reclutamiento de personal utilizadas por las empresas inscritas en la CCSM del sector Producción Tarapoto.

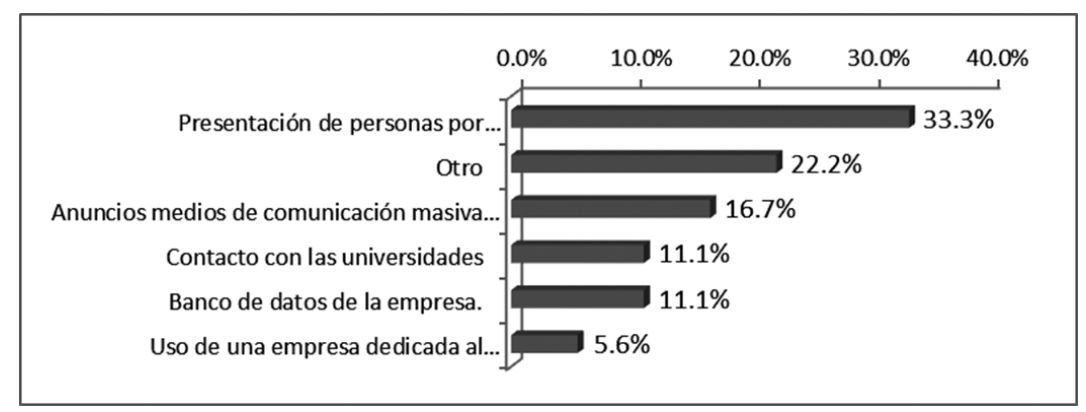

En la Figura 9 podemos observar que la técnica de reclutamiento de mayor aplicación en el sector Comercio es la presentación de personas por recomendación de los trabajadores con un 33\% del total, seguido de la publicación de carteles en los exteriores de la empresa, y la recomendación de familiares o amigos para la contratación de los mismos. Y en tercera opción la utilización de medios masivos de comunicación agrupados en otros con $22.2 \%$.

Figura 10. Técnicas de selección de personal utilizadas por las empresas inscritas en la CCSM del sector Producción en Tarapoto

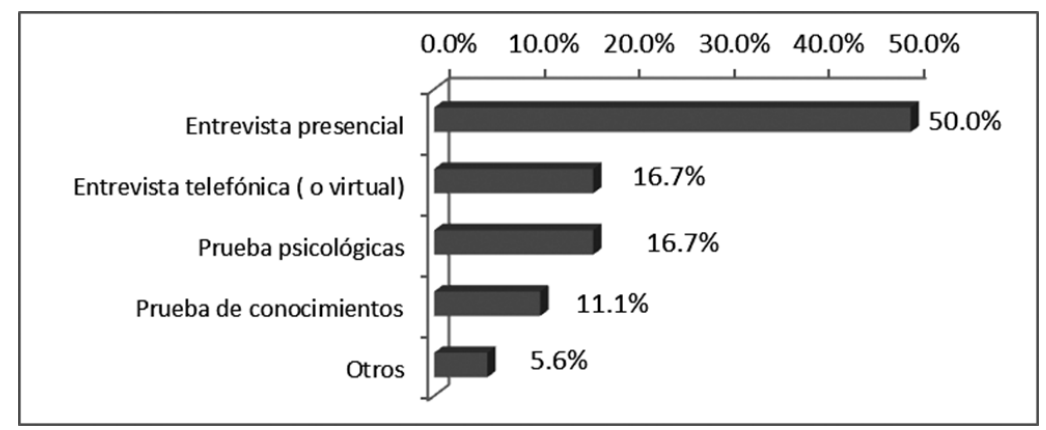


En cuanto a las técnicas de selección más usadas por el sector Producción en Tarapoto, encontramos a la entrevista personal con más del $50 \%$ del total, seguida de la entrevista telefónica, resaltando la técnica en sus dos modalidades, ambas con un $16.6 \%$, seguida de las pruebas psicológicas con el 11.1\%, como se puede apreciar en la Figura 10.

El sector servicios está conformado por los servicios técnicos, de vigilancia y seguridad, los servicios de gestión administrativa.

Transporte, banca y finanzas, médicos entre otros, $35.18 \%$ del total de la muestra del presente estudio

Tabla 7. Principales competencias valoradas por las empresas agrupadas en el sector Servicios.

\begin{tabular}{lcc}
\hline \multicolumn{1}{c}{ Competencias } & Recuento & Porcentaje \\
\hline Trabajo en equipo & 13 & 34.21 \\
Valores & 9 & 23.68 \\
Gestión Personal & 6 & 15.79 \\
Influencia & 6 & 15.79 \\
Cognitivas & 2 & 5.26 \\
Logro & 2 & 5.26 \\
Total & 38 & 100.00 \\
\hline
\end{tabular}

* Empresas solo del sector Servicios que cumplen con los requisitos de inclusión

Siendo que el sector servicios es el segundo agrupamiento más grande del estudio, como se presenta en la Tabla 7, la competencia de mayor valoración por parte de la empresa es el trabajo en equipo con un $34.21 \%$, seguido de los valores con un $23.68 \%$ y en tercer lugar las competencias de gestión personal referidas al autocontrol, la determinación y el temple e influencia $15.79 \%$.

Figura 11. Técnicas de reclutamiento de personal utilizadas por las empresas inscritas en la CCSM del sector Turismo en Tarapoto.

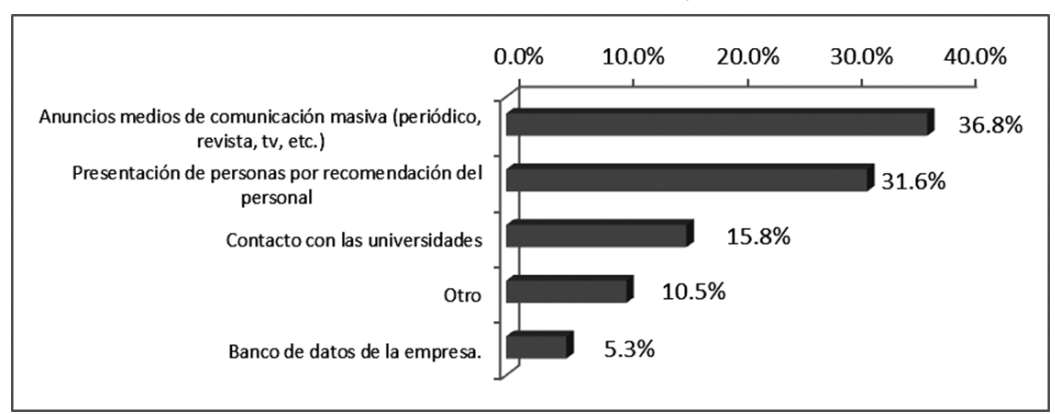


Las técnicas de reclutamiento de personal por medios masivos de comunicación son de mayor porcentaje con un $36.8 \%$ del total, seguido de la presentación de persona por parte del personal con un $31.6 \%$ y el contacto con las universidades con un $15.8 \%$, según la Figura 11.

Figura 12. Técnicas de selección de personal utilizadas por las empresas inscritas en la CCSM del sector Turismo en Tarapoto.

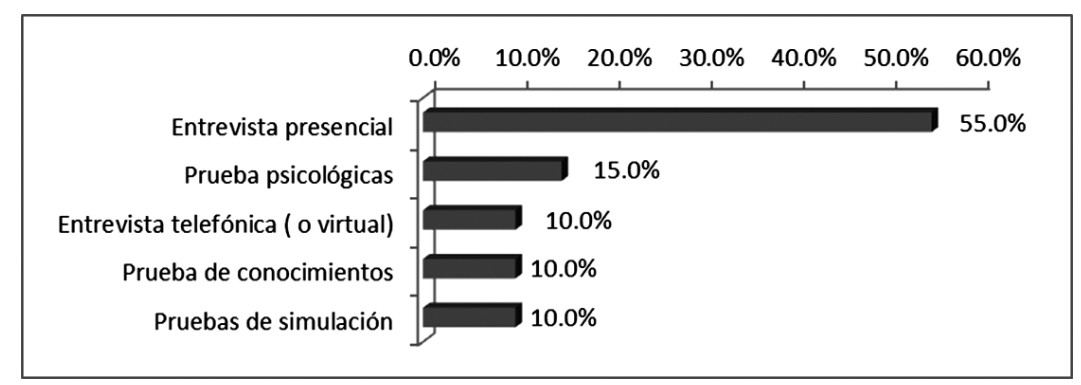

Como se aprecia en la figura 12 , la entrevista personal se mantiene como la técnica predominante en la selección, con un 55\% del total a las que se acompañan las pruebas psicológicas con un $15 \%$ y finalmente las pruebas de conocimientos, de simulación y entrevista virtual.

\section{Conclusión}

Las empresas en Tarapoto demandan más competencias de trabajo en equipo, siendo empatía, naturalidad y liderazgo las competencias más requeridas, repitiéndose esta conducta en todos los sectores que funcionan en la Cámara de Comercio de San Martín.

Siendo los valores, la segunda competencia más requerida como su segunda opción, se infiere la necesidad de colaboradores confiables que practiquen un criterio ético en el desarrollo de sus actividades.

En cuanto a las técnicas de reclutamiento, la más utilizada por las empresas en Tarapoto es la presentación de personas por recomendación del personal que actualmente labora en su empresa. Solo el sector turismo muestra mayor preferencia por la utilización de medios de comunicación masiva para su reclutamiento. Cabe señalar que las técnicas como el anuncio en la puerta de su negocio o empresa y las recomendaciones de amigos y conocidos son técnicas utilizadas con mayor incidencia en los sectores Comercio y Producción. 
La entrevista personal de modo presencial es la técnica predominante al momento de desarrollar el proceso de selección en todos los casos, dado que para el microempresario es la única manera que le es altamente confiable para corroborar los datos y las competencias de los candidatos.

Se puede observar que las pruebas psicológicas y cognitivas son aplicadas con mayor incidencia en los sectores turismo y servicios, las cuales en su mayoría son psicotécnicas. Al consultar sobre la persona que se encarga de la aplicación de las mismas, se pudo observar que de las 108 empresas del total de la muestra, solo una persona labora a tiempo completo y dos personas a tiempo parcial, mayormente en el sector servicios bancarios y educativo superior.

\section{Agradecimientos}

A los alumnos del curso de Gestión de Talento Humano, de la carrera de Administración, mención Gestión Empresarial, de la filial Tarapoto de la Universidad Peruana Unión, por su espíritu investigador, voluntad y denuedo que mostraron en el presente estudio.

Mario Manuel Siles Nates Universidad Peruana Unión email: mario.siles@upeu.edu.pe 
Mario Manuel Siles Nates

\section{Referencias}

Alles, M (2008). Dirección estratégica de recursos humanos. Buenos Aires: Granica.

Alles, M (2009). Diccionario de competencias. Buenos Aires: Granica.

Alles, M (2007). Selección por competencias. Buenos Aires: Granica.

Blanco, P .A (2008). Trabajadores competentes. España: Esic.

Chiavenato, I. (2009). Gestión del talento humano. Colombia: Macgraw-gill.

Flores, M. (2009). Principales competencias y modalidades de evaluación requeridas en procesos de selección de personal en el mercado profesional. PUC - Bolsa de Trabajo.

Hernandes, B (2007). Metodología de la investigación científica. Colombia: Macgraw-gill.

McClelland, D \& Watson, R. (1973). Power Motivation and Risk Talking Behavior, Journal of Personality.

Lévano, C. (2005). Elaboración de estadísticas de la microempresa. Lima: Ministerio de Trabajo 2005.

Luque, T. (1997). Investigación de marketing fundamentos. Barcelona: Ariel. 200-225.

Spencer, L (1993). Competence at Work, Model for Superior Performance, New York: John Wiley \& Sons inc.

Villarán, F (2007). El mundo de la pequeña empresa. Minsetour. 63-107. 